\title{
Two expedient 'one-pot' methods for synthesis of $\beta$-aryl- $\beta$-mercaptoketones over anhydrous potassium carbonate or amberlyst-15 catalyst
}

\author{
CHAYAN GUHA, RINA MONDAL, RAMMOHAN PAL and ASOK K MALLIK* \\ Department of Chemistry, Jadavpur University, Kolkata 700 032, India \\ e-mail: mallikak52@yahoo.co.in
}

MS received 29 April 2013; revised 24 August 2013; accepted 26 August 2013

\begin{abstract}
Two expedient one-pot methods have been developed for synthesis of $\beta$-aryl- $\beta$-mercaptoketones using acetophenones, benzaldehydes and thiols as starting materials. The methods involve microwave irradiation $(5 \mathrm{~min}$ ) of 1:1 mixtures of acetophenones and benzaldehydes over neutral alumina supported anhydrous potassium carbonate or amberlyst-15 in the first step, and that is followed by addition of thiol to the resulting material and keeping at room temperature for $1.5 \mathrm{~h}$.
\end{abstract}

Keywords. $\quad \beta$-aryl- $\beta$-mercaptoketones; anhydrous potassium carbonate; amberlyst-15; chalcones; thia-Michael addition.

\section{Introduction}

The chemistry of thia-Michael addition is being explored since a long period ${ }^{1}$ due to its versatile applications in biosynthesis ${ }^{2}$ and for obtaining compounds having biological activities such as tumour inhibitors, ${ }^{2}$ $\gamma$-secretase inhibitors, ${ }^{3}$ etc. In order to carry out such reactions, a good number of methodologies involving a variety of catalysts or catalytic systems have been developed so far; the important ones include different Lewis acids such as $\mathrm{CdI}_{2},{ }^{4} \mathrm{InBr}_{3},{ }^{5} \mathrm{Zn}\left(\mathrm{ClO}_{4}\right)_{2} \cdot 6 \mathrm{H}_{2} \mathrm{O},{ }^{6}$ molecular iodine, ${ }^{7,8} \mathrm{Bi}\left(\mathrm{NO}_{3}\right)_{3}{ }^{9}$ and $\mathrm{VO}(\mathrm{OTf})_{2},{ }^{10}$ etc. The process can also be catalysed by organic bases, both of synthetic and natural origin, ${ }^{11-16}$ complexes of different transition metals, ${ }^{17,18}$ ionic liquids, ${ }^{19-22}$ solid supports, ${ }^{23-26}$ phosphorus doped with other element or chemical species, ${ }^{27,28}$ polyethylene glycol, ${ }^{29}$ porphyrin rings, ${ }^{30}$ etc. However, the above mentioned methods involve some limitations such as use of expensive catalysts, long reaction time, etc. Besides, preparation of the Michael acceptors needs a separate step in all cases. These limitations led us to develop a new efficient methodology for a one-pot synthesis of $\beta$-aryl$\beta$-mercaptoketones (1), particularly because of recent success of our group and also of others in synthesis of 2,4-diarylthiochromans (2) utilizing them as useful intermediates (scheme 1). ${ }^{31,32}$

*For correspondence
A domino process usually refers to successive occurrence of different reactions in the same reaction vessel without separation or purification of reactive intermediates. ${ }^{33,34}$ Survey of literature showed a growing trend in utilization of domino sequential one-pot aldol-thiaMichael process for synthesis of some important $\beta$ aryl- $\beta$-mercaptoketones (1). However, reports on such processes are very limited. ${ }^{35,36}$ Herein, we report the development of two domino sequential one-pot aldolthia-Michael processes using inexpensive catalysts such as anhydrous $\mathrm{K}_{2} \mathrm{CO}_{3}$ or amberlyst-15 (a sulphonated polystyrene resin).

\section{Experimental}

Melting points were recorded on a Köfler block and are uncorrected. Infrared (IR) spectra were recorded on a Perkin Elmer Fourier Transform Infrared Spectrophotometer (Spectrum BX II) as $\mathrm{KBr}$ pellets. ${ }^{1} \mathrm{H}$ and ${ }^{13} \mathrm{C}$ Nuclear Magnetic Resonance (NMR) spectra were obtained in $\mathrm{CDCl}_{3}$ on a Bruker AV-300 $(300 \mathrm{MHz})$ spectrometer using tetramethylsilane as an internal standard. Mass spectrum was acquired on a Waters QTOF Micro YA263 Mass Spectrometer. Analytical samples were dried in vacuo at room temperature. Microanalytical data were recorded on two Perkin-Elmer 2400 Series II C, H, N analysers. An unmodified domestic household microwave oven (LG, DMO, Model No.-556P, 900 watt) equipped with inverter technology, which provides a realistic control 

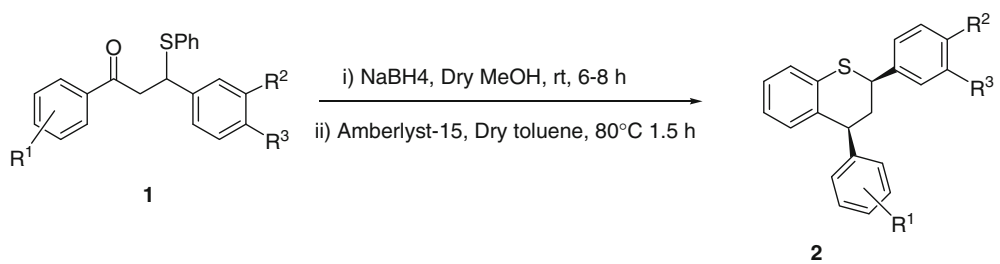

Scheme 1. Conversion of $\beta$-aryl- $\beta$-mercaptoketones (1) to cis-2,4-diarylthiochromans (2).

of the microwave power to the desired level (20-100\%) was used for microwave heating. Column chromatography was performed on silica gel (100-200 mesh) using petroleum ether $\left(60-80^{\circ} \mathrm{C}\right)$ and petroleum ether-ethyl acetate mixtures as eluents. Thin layer chromatography was done with silica gel $\mathrm{G}$.

\subsection{General procedure for synthesis of $\beta$-aryl- $\beta$-mercaptoketones (1)}

A mixture of acetophenone $(\mathbf{3}, 1 \mathrm{mmol})$ and aromatic aldehyde $(4,1 \mathrm{mmol})$ was thoroughly ground over neutral alumina $(4 \mathrm{~g})$ with added anhydrous $\mathrm{K}_{2} \mathrm{CO}_{3}$ $(1 \mathrm{mmol})$ or amberlyst-15 $(80 \mathrm{mg})$ and the resulting powder was subjected to microwave irradiation at $540 \mathrm{~W}$ for $5 \mathrm{~min}\left(120-125^{\circ} \mathrm{C}\right)$. After cooling the mass to room temperature, thiol $(\mathbf{5}, 1.3 \mathrm{mmol})$ was added and thoroughly mixed and then the mixture was kept at room temperature for $1.5 \mathrm{~h}$ under closed condition. The solid was then washed thoroughly with dichloromethane and the concentrate of the washings was subjected to column chromatography over silica gel using petroleum ether-ethyl acetate mixtures as eluents to get $\mathbf{1}$ in pure state.

The same method was followed for synthesis of $\mathbf{7}$ and 8 also.

\subsection{Analytical and spectral data of some selected $\beta$-aryl- $\beta$-mercaptoketones and related compounds}

2.2a 3-(4-Methylphenyl)-1-phenyl-3-phenylsulphanylpropan-1-one $\mathbf{l b}$ : Colourless crystals, IR (KBr) $v_{\max }=1668(\mathrm{C}=\mathrm{O}), 1597,1440,1329,1233,1176$, 1112, 994, 811, $728 \mathrm{~cm}^{-1}$; ${ }^{1} \mathrm{H}$ NMR $(300 \mathrm{MHz}$, $\left.\mathrm{CDCl}_{3}\right): \delta 2.28\left(\mathrm{~s}, 3 \mathrm{H},-\mathrm{CH}_{3}\right), 3.50-3.70(\mathrm{~m}, 2 \mathrm{H}$, $\left.-\mathrm{CH}_{2}\right), 4.94(\mathrm{t}, 1 \mathrm{H}, J=7.0 \mathrm{~Hz}, \mathrm{H}-2), 7.06(\mathrm{~d}, 2 \mathrm{H}$, $J=7.8 \mathrm{~Hz}), 7.22-7.26(\mathrm{~m}, 4 \mathrm{H}), 7.32-7.35(\mathrm{~m}, 2 \mathrm{H})$, $7.42(\mathrm{t}, 2 \mathrm{H}, J=7.2 \mathrm{~Hz}), 7.50-7.59(\mathrm{~m}, 2 \mathrm{H}), 7.87(\mathrm{~d}$, $2 \mathrm{H}, J=7.2 \mathrm{~Hz}$ ); Anal. Calcd. for $\mathrm{C}_{22} \mathrm{H}_{20} \mathrm{OS}$ (332.46): C, 79.48; H, 6.06. Found: C, 79.33; H, 6.22.

2.2b 3-(3-Nitrophenyl)-1-phenyl-3-phenylsulphanylpropan-1-one 1e: Light yellow crystals, IR (KBr) $v_{\max }=1671(\mathrm{C}=\mathrm{O}), 1531,1347,1224,987 \mathrm{~cm}^{-1} ;{ }^{1} \mathrm{H}$
NMR $\left(300 \mathrm{MHz}, \mathrm{CDCl}_{3}\right): \delta 3.68(\mathrm{~d}, 2 \mathrm{H}, J=7.2 \mathrm{~Hz}$, $\left.-\mathrm{CH}_{2}\right), 5.00(\mathrm{t}, 1 \mathrm{H}, J=7.2 \mathrm{~Hz}, \mathrm{H}-2), 7.23-7.34(\mathrm{~m}$, 5H), 7.37-7.48 (m, 3H), 7.55-7.63 (m, 2H), 7.88-7.91 $(\mathrm{m}, 2 \mathrm{H}), 8.02-8.07(\mathrm{~m}, 1 \mathrm{H}), 8.17(\mathrm{t}, 1 \mathrm{H}, J=2.4 \mathrm{~Hz})$; Anal. Calcd. for $\mathrm{C}_{21} \mathrm{H}_{17} \mathrm{NO}_{3} \mathrm{~S}$ (363.43): $\mathrm{C}, 69.40 ; \mathrm{H}$, 4.71 ; N, 3.85. Found: C, 69.22; H, 4.93; N, 3.98 .

2.2c 3-(4-Nitrophenyl)-1-phenyl-3-phenylsulphanylpropan-1-one 1f: Light yellow crystals, IR (KBr) $v_{\max }=1678(\mathrm{C}=\mathrm{O}), 1514,1346,1228,984$, $825 \mathrm{~cm}^{-1}$; ${ }^{1} \mathrm{H}$ NMR $\left(300 \mathrm{MHz}, \mathrm{CDCl}_{3}\right): \delta 3.67(\mathrm{~d}, 2 \mathrm{H}$, $\left.J=7.2 \mathrm{~Hz},-\mathrm{CH}_{2}\right), 4.97(\mathrm{t}, 1 \mathrm{H}, J=7.0 \mathrm{~Hz}, \mathrm{H}-2)$, 7.23-7.31 (m, 5H), 7.42-7.58 (m, 4H), 7.60-7.87 (m, $1 \mathrm{H}), 7.89(\mathrm{dd}, 2 \mathrm{H}, J=8.1$ and $1.2 \mathrm{~Hz}), 8.08(\mathrm{~d}, 2 \mathrm{H}$, $J=8.7 \mathrm{~Hz}$ ); Anal. Calcd. for $\mathrm{C}_{21} \mathrm{H}_{17} \mathrm{NO}_{3} \mathrm{~S}(363.43)$ : C, 69.40; H, 4.71; N, 3.85. Found: C, 69.52; H, 4.63; $\mathrm{N}, 4.01$.

2.2d 3-(4-N,N-Dimethylphenyl)-1-phenyl-3-phenylsulphanylpropan-1-one 1g: Yellow crystals, IR (KBr) $v_{\max }=1679(\mathrm{C}=\mathrm{O}), 1525,1360,1229,948,806$, $756 \mathrm{~cm}^{-1} ;{ }^{1} \mathrm{H}$ NMR $\left(300 \mathrm{MHz}, \mathrm{CDCl}_{3}\right): \delta 2.91(\mathrm{~s}, 6 \mathrm{H}$, $\left.-\mathrm{N}\left(\mathrm{CH}_{3}\right)_{2}\right), 3.48-3.69\left(\mathrm{~m}, 2 \mathrm{H},-\mathrm{CH}_{2}\right), 4.93(\mathrm{dd}, 1 \mathrm{H}$, $J=8.4$ and $5.8 \mathrm{~Hz}, \mathrm{H}-2), 6.67(\mathrm{~d}, 2 \mathrm{H}, J=7.8 \mathrm{~Hz})$, $7.17-7.26(\mathrm{~m}, 4 \mathrm{H}), 7.35(\mathrm{dd}, 2 \mathrm{H}, J=7.8$ and $1.8 \mathrm{~Hz})$, $7.41(\mathrm{t}, 2 \mathrm{H}, J=7.5 \mathrm{~Hz}), 7.53(\mathrm{t}, 2 \mathrm{H}, J=6.9 \mathrm{~Hz})$, $7.86(\mathrm{~d}, 2 \mathrm{H}, J=7.2 \mathrm{~Hz})$; Anal. Calcd. for $\mathrm{C}_{23} \mathrm{H}_{23} \mathrm{NOS}$ (361.50): C, 76.42; H, 6.41; N, 3.87. Found: C, 76.18; $\mathrm{H}, 6.20 ; \mathrm{N}, 3.91$.

2.2e 3-(3,4-Methylenedioxyphenyl)-1-phenyl-3-phenylsulphanylpropan-1-one 1h: Colourless crystals, IR $(\mathrm{KBr}) v_{\max }=1678(\mathrm{C}=\mathrm{O}), 1596,1581,1489,1255$, $1038,934,744 \mathrm{~cm}^{-1} ;{ }^{1} \mathrm{H}$ NMR $\left(300 \mathrm{MHz}, \mathrm{CDCl}_{3}\right): \delta$ $3.48-3.67\left(\mathrm{~m}, 2 \mathrm{H},-\mathrm{CH}_{2}\right), 4.92(\mathrm{t}, 1 \mathrm{H}, J=6.8 \mathrm{~Hz}, \mathrm{H}-$ 2), $5.89\left(\mathrm{~s}, 2 \mathrm{H},-\mathrm{CH}_{2}-\right) 6.65(\mathrm{~d}, 1 \mathrm{H}, J=7.8 \mathrm{~Hz}), 6.77$ $(\mathrm{d}, 1 \mathrm{H}, J=7.8 \mathrm{~Hz}), 6.92(\mathrm{~s}, 1 \mathrm{H}) 7.24(\mathrm{~d}, 3 \mathrm{H}, J=$ $5.4 \mathrm{~Hz}), 7.35-7.45(\mathrm{~m}, 4 \mathrm{H}), 7.53(\mathrm{~d}, 1 \mathrm{H}, J=6.6 \mathrm{~Hz})$, $7.88(\mathrm{~d}, 2 \mathrm{H}, J=7.2 \mathrm{~Hz})$; Anal. Calcd. for $\mathrm{C}_{22} \mathrm{H}_{18} \mathrm{O}_{3} \mathrm{~S}$ (362.44): C, 72.90; H, 5.01. Found: C, 72.61; H, 4.82.

$2.2 \mathrm{f}$ 3-Phenyl-1-(4-methylphenyl)-3-phenylsulphanylpropan-1-one 1i: Colourless crystals, IR (KBr) $v_{\max }=1674(\mathrm{C}=\mathrm{O}), 1605,1440,1338,1231,1180$, 
976, $812 \mathrm{~cm}^{-1} ;{ }^{1} \mathrm{H}$ NMR $\left(300 \mathrm{MHz}, \mathrm{CDCl}_{3}\right): \delta 2.39$ $\left(\mathrm{s}, 3 \mathrm{H},-\mathrm{CH}_{3}\right), 3.56-3.61\left(\mathrm{~m}, 2 \mathrm{H},-\mathrm{CH}_{2}\right), 4.94(\mathrm{dd}, 1 \mathrm{H}$, $J=7.8$ and $6.3 \mathrm{~Hz}, \mathrm{H}-2), 7.17-7.22(\mathrm{~m}, 4 \mathrm{H}), 7.23$ $(\mathrm{d}, 2 \mathrm{H}, J=1.8 \mathrm{~Hz}), 7.26(\mathrm{~s}, 2 \mathrm{H}), 7.30-7.34(\mathrm{~m}, 4 \mathrm{H})$, $7.77(\mathrm{~d}, 2 \mathrm{H}, J=8.1 \mathrm{~Hz})$; Anal. Calcd. for $\mathrm{C}_{22} \mathrm{H}_{20} \mathrm{OS}$ (332.46): C, 79.48; H, 6.06. Found: C, 79.64; H, 6.22.

$2.2 \mathrm{~g}$ 3-(4-Methoxyphenyl)-1-(4-methylphenyl)-3-phenylsulphanylpropan-1-one 1j: Colourless crystals, IR $(\mathrm{KBr}) v_{\max }=1676(\mathrm{C}=\mathrm{O}), 1597,1460,1338,1251$, 1036, 976, $812 \mathrm{~cm}^{-1} ;{ }^{1} \mathrm{H}$ NMR $\left(300 \mathrm{MHz}, \mathrm{CDCl}_{3}\right): \delta$ $2.39\left(\mathrm{~s}, 3 \mathrm{H},-\mathrm{CH}_{3}\right), 3.52-3.60\left(\mathrm{~m}, 2 \mathrm{H},-\mathrm{CH}_{2}\right), 3.75(\mathrm{~s}$, $\left.3 \mathrm{H},-\mathrm{OCH}_{3}\right), 4.92(\mathrm{dd}, 1 \mathrm{H}, J=8.3$ and $5.8 \mathrm{~Hz}, \mathrm{H}-$ 2), $6.78(\mathrm{~d}, 2 \mathrm{H}, J=8.4 \mathrm{~Hz}), 7.20-7.34(\mathrm{~m}, 9 \mathrm{H}), 7.77$ $(\mathrm{d}, 2 \mathrm{H}, J=8.1 \mathrm{~Hz}) ;{ }^{13} \mathrm{C}$ NMR $\left(75 \mathrm{MHz}, \mathrm{CDCl}_{3}\right)$ : 21.6, 44.7, 47.7, 55.2, 113.8, 127.4, 128.2, 128.8, 129.3, 132.6, 133.2, 134.3, 134.5, 144.1, 158.7, 196.8; TOF $\mathrm{MS} \mathrm{ES}^{+}(\mathrm{M}+\mathrm{Na})^{+}$: Calcd. 385.1238. Found 385.1237; Anal. Calcd. for $\mathrm{C}_{23} \mathrm{H}_{22} \mathrm{O}_{2} \mathrm{~S}$ (362.48): C, 76.21; H, 6.12. Found: C, 75.98; H, 5.94.

2.2h 3-(4-Chlorophenyl)-1-(4-methylphenyl)-3-phenylsulphanylpropan-1-one 1k: Colourless crystals, IR $(\mathrm{KBr}) v_{\max }=1656(\mathrm{C}=\mathrm{O}), 1564,1492,1332,1184$, 1012, 988, $817 \mathrm{~cm}^{-1} ;{ }^{1} \mathrm{H}$ NMR $\left(300 \mathrm{MHz}, \mathrm{CDCl}_{3}\right): \delta$ $2.39\left(\mathrm{~s}, 3 \mathrm{H},-\mathrm{CH}_{3}\right), 3.48-3.63\left(\mathrm{~m}, 2 \mathrm{H},-\mathrm{CH}_{2}\right), 4.90(\mathrm{t}$, $1 \mathrm{H}, J=7.1 \mathrm{~Hz}, \mathrm{H}-2), 7.18-7.31(\mathrm{~m}, 11 \mathrm{H}), 7.77(\mathrm{~d}, 2 \mathrm{H}$, $J=8.1 \mathrm{~Hz}$ ); Anal. Calcd. for $\mathrm{C}_{22} \mathrm{H}_{19} \mathrm{ClOS}$ (366.90): C, 72.02; H, 5.22. Found: C, 72.21; H, 4.98.

2.2i 3-Phenyl-1-(4-methoxyphenyl)-3-phenylsulphanylpropan-1-one 11: Colourless crystals, IR (KBr) $v_{\max }=1670(\mathrm{C}=\mathrm{O}), 1601,1573,1423,1338$, $1233,1173,1024,984,845 \mathrm{~cm}^{-1} ;{ }^{1} \mathrm{H}$ NMR $(300 \mathrm{MHz}$, $\left.\mathrm{CDCl}_{3}\right): \delta$ 3.47-3.65 (m, 2H, $\left.-\mathrm{CH}_{2}\right), 3.85(\mathrm{~s}, 3 \mathrm{H}$, $\left.-\mathrm{OCH}_{3}\right), 4.95(\mathrm{dd}, 1 \mathrm{H}, J=7.8$ and $6.0 \mathrm{~Hz}, \mathrm{H}-2), 6.89$ $(\mathrm{d}, 2 \mathrm{H}, J=8.7 \mathrm{~Hz}), 7.15-7.34(\mathrm{~m}, 10 \mathrm{H}), 7.87(\mathrm{~d}, 2 \mathrm{H}$, $J=6.9 \mathrm{~Hz}$ ); Anal. Calcd. for $\mathrm{C}_{22} \mathrm{H}_{20} \mathrm{ClO}_{2} \mathrm{~S}$ (348.46): C, 75.83; H, 5.79. Found: C, 75.91; H, 5.84.

2.2j 3-(4-Methoxyphenyl)-1-(4-methoxyphenyl)-3phenylsulphanylpropan-1-one $1 \mathrm{~m}$ : Colourless crystals, IR (KBr) $v_{\max }=1672(\mathrm{C}=\mathrm{O}), 1607,1515$, 1256, 1179, 1030, 822, $733 \mathrm{~cm}^{-1} ;{ }^{1} \mathrm{H}$ NMR $(300 \mathrm{MHz}$, $\left.\mathrm{CDCl}_{3}\right): \delta 3.44-3.62\left(\mathrm{~m}, 2 \mathrm{H},-\mathrm{CH}_{2}\right), 3.75(\mathrm{~s}, 3 \mathrm{H}$, $\left.-\mathrm{OCH}_{3}\right), 3.84\left(\mathrm{~s}, 3 \mathrm{H},-\mathrm{OCH}_{3}\right), 4.94(\mathrm{dd}, 1 \mathrm{H}, J=8.1$ and $6.0 \mathrm{~Hz}, \mathrm{H}-2), 6.77(\mathrm{~d}, 2 \mathrm{H}, J=8.7 \mathrm{~Hz}), 6.89(\mathrm{~d}$, $2 \mathrm{H}, J=8.7 \mathrm{~Hz}), 7.18-7.35(\mathrm{~m}, 7 \mathrm{H}), 7.85(\mathrm{~d}, 2 \mathrm{H}$, $J=8.7 \mathrm{~Hz}) ;{ }^{13} \mathrm{C} \mathrm{NMR}\left(75 \mathrm{MHz}, \mathrm{CDCl}_{3}\right): 44.4,47.8$, $55.2,55.5,113.7,113.8,127.3,128.8,128.9,129.9$, 130.4, 132.6, 133.3, 134.6, 158.7, 163.6, 195.6; TOF $\mathrm{MS} \mathrm{ES}^{+}(\mathrm{M}+\mathrm{Na})^{+}$: Calcd. 401.1187. Found 401.1188;
Anal. Calcd. for $\mathrm{C}_{23} \mathrm{H}_{22} \mathrm{O}_{3} \mathrm{~S}$ (378.48): C, 72.99; H, 5.86. Found: C, 72.71; H, 5.74.

2.2k 3-(4-Chlorophenyl)-1-(4-methoxyphenyl)-3-phenylsulphanylpropan-1-one 1n: Colourless crystals, IR $(\mathrm{KBr}) v_{\max }=1603(\mathrm{C}=\mathrm{O}), 1574,1492,1328$, $1256,1176,1025,982,818 \mathrm{~cm}^{-1} ;{ }^{1} \mathrm{H}$ NMR $(300 \mathrm{MHz}$, $\left.\mathrm{CDCl}_{3}\right): \delta 3.51-3.55\left(\mathrm{~m}, 2 \mathrm{H},-\mathrm{CH}_{2}\right), 3.85(\mathrm{~s}, 3 \mathrm{H}$, $\left.-\mathrm{OCH}_{3}\right), 4.90(\mathrm{t}, 1 \mathrm{H}, J=7.0 \mathrm{~Hz}, \mathrm{H}-2), 6.90(\mathrm{~d}, 2 \mathrm{H}$, $J=8.4 \mathrm{~Hz}), 7.18-7.31(\mathrm{~m}, 9 \mathrm{H}), 7.86(\mathrm{~d}, 2 \mathrm{H}, J=$ 7.8 Hz); Anal. Calcd. for $\mathrm{C}_{22} \mathrm{H}_{19} \mathrm{ClO}_{2} \mathrm{~S}$ (382.90): C, 69.01; H, 5.00. Found: C, 69.22; H, 4.83.

2.21 3-(4-Chlorophenyl)-1-phenyl-3-(4-methylphenylsulphanyl)propan-1-one 1o: Colourless crystals, IR $(\mathrm{KBr}) v_{\max }=1678(\mathrm{C}=\mathrm{O}), 1492,1325,1226,1093$, $814 \mathrm{~cm}^{-1} ;{ }^{1} \mathrm{H}$ NMR $\left(300 \mathrm{MHz}, \mathrm{CDCl}_{3}\right): \delta 2.30(\mathrm{~s}, 3 \mathrm{H}$, $\left.-\mathrm{CH}_{3}\right) 3.50-3.65\left(\mathrm{~m}, 2 \mathrm{H},-\mathrm{CH}_{2}\right), 4.83(\mathrm{t}, 1 \mathrm{H}, J=$ $7.0 \mathrm{~Hz}, \mathrm{H}-2), 7.04(\mathrm{~d}, 2 \mathrm{H}, J=7.8 \mathrm{~Hz}), 7.13-7.26$ $(\mathrm{m}, 6 \mathrm{H}), 7.43$ (t, 2H, $J=7.5 \mathrm{~Hz}.), 7.55$ (br. t, $1 \mathrm{H}$, $J=7.3 \mathrm{~Hz}$ ), 7.87 (br. d, $2 \mathrm{H}, J=7.2 \mathrm{~Hz}$ ); ${ }^{13} \mathrm{C} \mathrm{NMR}$ (75 MHz, $\mathrm{CDCl}_{3}$ ): 21.1, 44.5, 48.1, 128.1, 128.5, 128.7, 129.2, 129.8, 129.9, 132.9, 133.4, 133.7, 136.7, 138.1, 140.0, 196.8; Anal. Calcd. for $\mathrm{C}_{22} \mathrm{H}_{19} \mathrm{ClOS}$ (366.08): C, 72.02; H, 5.22. Found: C, 72.25; H, 5.36.

2.2m 3-(4-Nitrophenyl)-1-(4-chlorophenyl)-3-(4methylphenylsulphanyl)propan-1-one 1p: Colourless crystals, IR $(\mathrm{KBr}) v_{\max }=1686(\mathrm{C}=\mathrm{O}), 1514,1344$, $1219,985,822 \mathrm{~cm}^{-1} ;{ }^{1} \mathrm{H}$ NMR $\left(300 \mathrm{MHz}, \mathrm{CDCl}_{3}\right): \delta$ $2.30\left(\mathrm{~s}, 3 \mathrm{H},-\mathrm{CH}_{3}\right) 3.59-3.62\left(\mathrm{~m}, 2 \mathrm{H},-\mathrm{CH}_{2}\right), 4.87(\mathrm{t}$, $1 \mathrm{H}, J=6.9 \mathrm{~Hz}, \mathrm{H}-2), 7.05(\mathrm{~d}, 2 \mathrm{H}, J=7.8 \mathrm{~Hz})$, $7.17(\mathrm{~d}, 2 \mathrm{H}, J=7.8 \mathrm{~Hz}), 7.26(\mathrm{~s}, 1 \mathrm{H}), 7.42(\mathrm{t}, 3 \mathrm{H}$, $J=7.5 \mathrm{~Hz}), 7.83(\mathrm{~d}, 2 \mathrm{H}, J=8.4 \mathrm{~Hz}), 8.09(\mathrm{~d}, 2 \mathrm{H}$, $J=8.4 \mathrm{~Hz}$ ); Anal. Calcd. for $\mathrm{C}_{22} \mathrm{H}_{18} \mathrm{ClNO}_{3} \mathrm{~S}$ (411.07): C, 64.15; H, 4.40. Found: C, 63.90; H, 4.31.

2.2n 3-Phenyl-1-(3-nitrophenyl)-3-phenylsulphanylpropan-1-one 1s: Light yellow crystals, IR ( $\mathrm{KBr}$ ) $v_{\max }=1674(\mathrm{C}=\mathrm{O}), 1536,1349,1236,987 \mathrm{~cm}^{-1} ;{ }^{1} \mathrm{H}$ NMR $\left(300 \mathrm{MHz}, \mathrm{CDCl}_{3}\right): \delta 3.57-3.73\left(\mathrm{~m}, 2 \mathrm{H},-\mathrm{CH}_{2}\right)$, $4.94(\mathrm{t}, 1 \mathrm{H}, J=7.0 \mathrm{~Hz}, \mathrm{H}-2), 7.21-7.39(\mathrm{~m}, 10 \mathrm{H})$, $7.64(\mathrm{t}, 1 \mathrm{H}, J=8.0 \mathrm{~Hz}), 8.18(\mathrm{dd}, 1 \mathrm{H}, J=7.8$ and $0.9 \mathrm{~Hz}), 8.38(\mathrm{dt}, 1 \mathrm{H}, J=8.1$ and $0.9 \mathrm{~Hz}), 8.67(\mathrm{~s}$, $1 \mathrm{H})$; Anal. Calcd. for $\mathrm{C}_{21} \mathrm{H}_{17} \mathrm{NO}_{3} \mathrm{~S}$ (363.43): C, 69.40; H, 4.71; N, 3.85. Found: C, 69.24; H, 4.95; N, 4.02.

2.2o (E)-1,5-diphenyl-3-(phenylthio)pent-4-en-1-one 7: Colourless crystals, mp: $102-104^{\circ} \mathrm{C}$. IR (KBr) $v_{\max }=1672(\mathrm{C}=\mathrm{O}), 1607,1515,1256,1179,1030$, $822,733 \mathrm{~cm}^{-1} .{ }^{1} \mathrm{H}$ NMR $\left(300 \mathrm{MHz}, \mathrm{CDCl}_{3}\right): \delta 3.40$ $3.43\left(\mathrm{~m}, 2 \mathrm{H}, \mathrm{CH}_{2}\right), 4.48(\mathrm{~m}, 1 \mathrm{H},>\mathrm{CH}-\mathrm{S}-), 6.16$ 
Table 1. Optimization of reaction conditions for generation of chalcone (6a) by variation of MW power and irradiation time.

\begin{tabular}{lcccc}
\hline & & \multicolumn{2}{c}{ Yield $^{\mathrm{a}}$ of 6 (\%) } \\
\cline { 4 - 5 } Entry & Power (W) & Time (min) & Anh. $\mathrm{K}_{2} \mathrm{CO}_{3}$ & Amberlyst-15 \\
\hline 1 & 180 & 10 & nil & Nil \\
2 & 360 & 3 & 5 & Trace \\
3 & 360 & 5 & 27 & 16 \\
4 & 360 & 7 & 43 & 30 \\
5 & 540 & 3 & 85 & 72 \\
6 & 540 & 5 & 97 & 85 \\
7 & 540 & 7 & 97 & 85 \\
8 & 720 & 3 & Reaction mixture charred \\
\hline
\end{tabular}

${ }^{a}$ Isolated yield for this and all the subsequent tables

$(\mathrm{dd}, 1 \mathrm{H}, J=15.7$ and $8.0 \mathrm{~Hz}, \mathrm{H}-4), 6.30(\mathrm{~d}, 1 \mathrm{H}$, $J=15.6 \mathrm{~Hz}, \mathrm{H}-5), 7.19-7.33(\mathrm{~m}, 8 \mathrm{H}$, Ar-H), 7.44$7.60(\mathrm{~m}, 5 \mathrm{H}, \mathrm{Ar}-\mathrm{H}), 7.92-7.94(\mathrm{~m}, 2 \mathrm{H}$, ortho-protons of - $\mathrm{COPh}$ ) Anal. Calcd. for $\mathrm{C}_{23} \mathrm{H}_{20} \mathrm{OS}$ (344.47): C, 80.19; H, 5.85; Found: C, 80.04; H, 6.02.

$2.2 \mathrm{p}$ Diastereomers of 1,3,8,10-tetraaryl-4,7-dithiadecan-1,10-dione 8: Colourless crystals, melting range $118-126^{\circ} \mathrm{C} ;{ }^{1} \mathrm{H}$ NMR $\left(300 \mathrm{MHz}, \mathrm{CDCl}_{3}\right): \delta$ 2.40-2.50 (m, 4H, $-\mathrm{S}_{-} \mathrm{CH}_{2}-\mathrm{CH}_{2}-\mathrm{S}$ - of the major isomer), $2.60-2.68\left(\mathrm{~m}, \sim 1.33 \mathrm{H},-\mathrm{S}-\mathrm{CH}_{2}-\mathrm{CH}_{2}-\mathrm{S}\right.$ - of the minor isomer), $3.45\left(\mathrm{~d}, 4 \mathrm{H}, J=6.9 \mathrm{~Hz},-\mathrm{CO}-\underline{\mathrm{C}}_{2}-\right.$ $\mathrm{CH}<$ of the major isomer), $3.51(\mathrm{~d}, \sim 1 \mathrm{H}, J=6.9 \mathrm{~Hz}$, $-\mathrm{CO}-\mathrm{C}_{2}-\mathrm{CH}<$ of the minor isomer), 4.46 (br. $\mathrm{t}, 2 \mathrm{H}$, $J=6.9 \mathrm{~Hz},-\mathrm{CO}-\mathrm{CH}_{2}-\mathrm{CH}<$ of the major isomer), 4.58 (br. t, $\sim 0.5 \mathrm{H}, J=6.9 \mathrm{~Hz},-\mathrm{CO}-\mathrm{CH}_{2}-\mathrm{C} \underline{\mathrm{H}}<$ of the minor isomer), 7.23-7.58 (m, $\sim 12.5 \mathrm{H}, \mathrm{Ar}-\mathrm{H}$ of both the isomers), 7.86-7.89 ( $\sim 5 \mathrm{H}$, ortho protons of $-\mathrm{COC}_{6} \mathrm{H}_{5}$ of both the isomers).

\section{Results and discussion}

Our endeavour for synthesis of $\beta$-aryl- $\beta$ mercaptoketones (1) started with searching of a suitable condition for a three-component reaction. Thus, when an equimolar mixture of acetophenone (3a),

Table 2. Optimization of reaction conditions for generation of $\mathbf{1 a}$ by use of different alkali metal carbonates ${ }^{\mathrm{a}}$.

\begin{tabular}{lcc}
\hline Entry & Metal carbonate & Yield (\%) \\
\hline 1 & $\mathrm{Na}_{2} \mathrm{CO}_{3}$ & 81 \\
2 & $\mathrm{~K}_{2} \mathrm{CO}_{3}$ & 94 \\
3 & $\mathrm{Cs}_{2} \mathrm{CO}_{3}$ & 96 \\
\hline
\end{tabular}

${ }^{\mathrm{a}} \mathrm{Mol}$ ratio of substrate to metal carbonate $=1: 1$ (yield optimized by use of $\mathrm{K}_{2} \mathrm{CO}_{3}$ ); amount of neutral alumina (solid support) $=4 \mathrm{~g} / \mathrm{mmol}$ of substrate (yield optimized) benzaldehyde (4a) and thiophenol (5a) was subjected to microwave irradiation over neutral alumina supported potassium carbonate or amberlyst- 15 , instead of the desired compound 1a, the chalcone $\mathbf{6 a}$ was formed. The strong characteristic smell of thiophenol obtained by opening the microwave oven after operation clearly indicated that failure of the expected reaction was due to vaporization of thiophenol from the reaction mixture. We, therefore, chose the strategy of generating chalcones (6) first and then allowing them to react with thiols in the same pot under a milder condition. It may be mentioned here that microwave-assisted Michael reactions over anhydrous $\mathrm{K}_{2} \mathrm{CO}_{3}$ are known in literature. ${ }^{37}$ The reaction condition for generation of chalcones (6) by microwave irradiation over neutral alumina supported anhydrous $\mathrm{K}_{2} \mathrm{CO}_{3}{ }^{38,39}$ or amberlyst$15^{40}$ was freshly optimized in the present study applying various microwave (MW) powers and irradiation times (table 1). Screening of different alkali metal carbonates and sulphonic acids for their catalytic activities was also done in this connection (tables 2 and 3). When MW irradiation was done over only neutral alumina, formation of only $\mathbf{6 a}$ (yield 52\%) was observed.

The results presented in table 1 clearly showed that irradiation at $540 \mathrm{~W}$ for $5 \mathrm{~min}$ was the optimum condition for the reaction. The choice of potassium carbonate

Table 3. Optimization of reaction conditions for generation of $1 \mathbf{a}$ by use of different sulphonic acids ${ }^{\mathrm{a}}$.

\begin{tabular}{lcc}
\hline Entry & Sulphonic acid & Yield (\%) \\
\hline 1 & Amberlyst-15 & 82 \\
2 & Camphor sulphonic acid & 11 \\
3 & $p$-TsOH & 52 \\
\hline
\end{tabular}

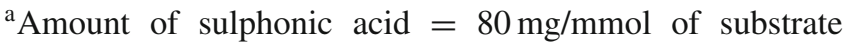
(yield optimized by use of amberlyst-15); amount of neutral alumina (solid support) $=4 \mathrm{~g} / \mathrm{mmol}$ of substrate (yield optimized) 


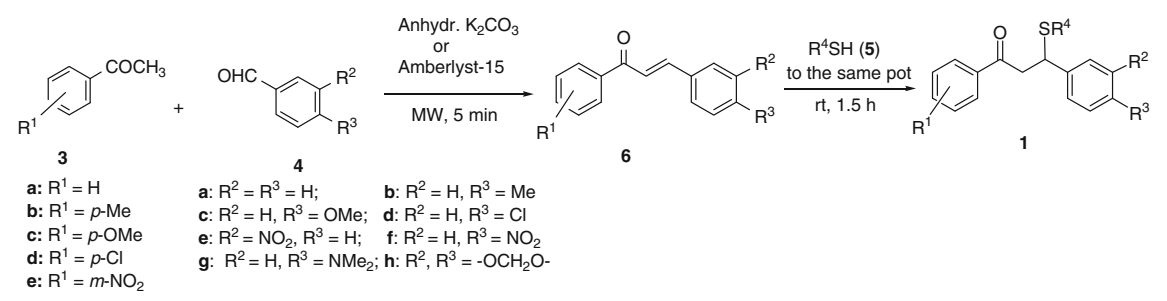

Scheme 2. Strategy for two-step one-pot synthesis of $\beta$-aryl- $\beta$-mercaptoketones (1).

over other alkali metal carbonates or amberlyst- 15 over other sulphonic acids was justified by the optimum yield and cost effectiveness (tables 2 and 3, respectively).

In the chosen strategy, a reaction mixture using $\mathbf{3 a}$ and $\mathbf{4 a}$ as starting materials (1:1 mol ratio) was allowed to cool to room temperature after microwave irradiation over anh. $\mathrm{K}_{2} \mathrm{CO}_{3}$ or amberlyst- 15 and then thiophenol (5a) (1.3 mol ratio) was added to the same pot and allowed to stand for $1.5 \mathrm{~h}$ (scheme 2). TLC examination at that point indicated the formation of 1a in high yield along with a trace amount of 6a. From this mixture, pure 1a could be obtained through rapid column chromatography.

This protocol was successfully employed for synthesis of 18 other $\beta$-aryl- $\beta$-mercaptoketones (1b-s) by using appropriate combinations of acetophenones (3), benzaldehydes (4) and thiols (5) and also for synthesis of $\mathbf{7}$ by use of acetophenone (3a) cinnamaldehyde and thiophenol (table 4). It is noteworthy that in

Table 4. Results of one-pot synthesis of $\beta$-aryl- $\beta$-mercaptoketones (1) and a related compound (7) .

Melting point $\left({ }^{\circ} \mathrm{C}\right)$
[Lit.]


Table 4. (continued)

\begin{tabular}{|c|c|c|c|c|}
\hline \multirow[b]{2}{*}{ Entry } & \multirow[b]{2}{*}{ Product (1/7) } & \multicolumn{2}{|c|}{ Yield (\%) } & \multirow{2}{*}{$\begin{array}{c}\text { Melting point }\left({ }^{\circ} \mathrm{C}\right) \\
\text { [Lit.] }\end{array}$} \\
\hline & & Anhydr. $\mathrm{K}_{2} \mathrm{CO}_{3}$ & Amberlyst-15 & \\
\hline 8 & $1 \mathrm{~h}$ & 87 & 79 & $66-68$ \\
\hline 9 & $1 \mathrm{i}$ & 93 & 80 & $60-62$ \\
\hline 10 & $1 \mathrm{j}$ & 88 & 78 & $112-114$ \\
\hline 11 & $1 \mathrm{k}$ & 94 & 81 & $90-92$ \\
\hline 12 & 11 & 91 & 80 & $118-120$ \\
\hline 13 & $1 \mathrm{~m}$ & 89 & 78 & $110-112$ \\
\hline 14 & in & 90 & 79 & $96-98$ \\
\hline 15 & 10 & 83 & 72 & $118-120$ \\
\hline 16 & 10 & 85 & 73 & $126-128$ \\
\hline 17 & & 88 & 75 & $60-62[59-62]^{8}$ \\
\hline
\end{tabular}


Table 4. (continued)

Yield (\%)
Anhydr. $\mathrm{K}_{2} \mathrm{CO}_{3} \quad$ Amberlyst-15 $\begin{gathered}\text { Melting point }\left({ }^{\circ} \mathrm{C}\right) \\ \text { [Lit.] }\end{gathered}$

${ }^{a}$ The ${ }^{1} \mathrm{H}$ NMR spectral features of the product in this case (vide Experimental) led to the elimination of the alternative structure $\mathbf{7}^{\prime}$.<smiles>O=C(/C=C/CC(c1ccccc1)c1ccccc1)c1ccccc1</smiles>

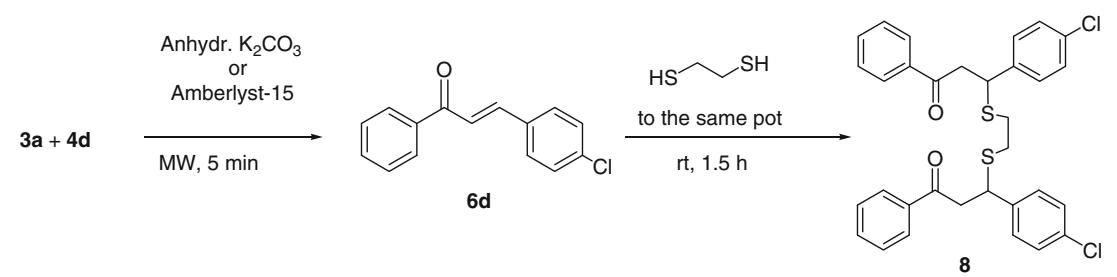

Scheme 3. Synthesis of 1,3,8,10-tetraaryl-4,7-dithia-decan-1,10-dione (8).

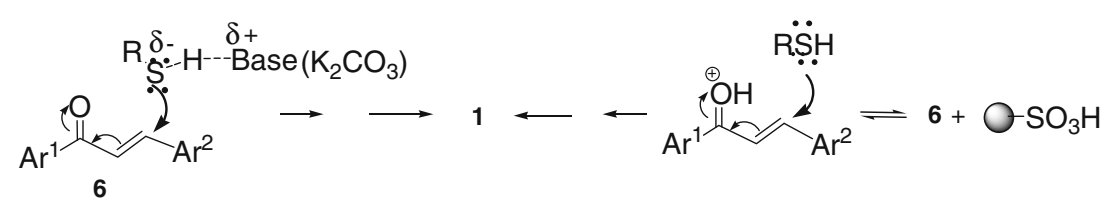

Scheme 4. Plausible mechanism of formation of $\beta$-aryl- $\beta$-mercaptoketones (1).

the latter case, $\beta$-position of the intermediate $E$ cinnamylideneacetophenone is selectively attacked by thiophenol over $\delta$-position.

The mentioned methodology was then extended to the combination of $\mathbf{3 a}, \mathbf{4 d}$ and ethane-1,2-dithiol (mol ratio: 1:1:0.6), when a 1:4 (approx.) mixture of the diastereomers of $\mathbf{8}$ was obtained in $63 \%$ yield. However, attempted separation of these diastereomers by column chromatography over silica gel did not meet with success (scheme 3).

Regarding the mechanistic aspects of the thiaMichael addition, it may be pointed out that in the $\mathrm{K}_{2} \mathrm{CO}_{3}$-catalysed process, the thiols are activated, while in the amberlyst-15-catalysed process, the $\alpha, \beta$ unsaturated ketones are activated to make the addition effective (scheme 4).

\section{Conclusion}

We have developed a very simple protocol for rapid, efficient and one-pot synthesis of $\beta$-aryl- $\beta$ mercaptoketones by use of tandem reactions on common inexpensive catalysts such as anhydrous $\mathrm{K}_{2} \mathrm{CO}_{3}$ 
and amberlyst- 15 . Such processes are a matter of current interest in the literature. ${ }^{36}$

\section{Supplementary information}

The electronic supplementary material contains ${ }^{1} \mathrm{H}$ NMR, ${ }^{13} \mathrm{C}$ NMR and mass spectra of a number of compounds of the series $\mathbf{1}, \mathbf{7}$ and $\mathbf{8}$, which can be seen in www.ias.ac.in/chemsci.

\section{Acknowledgements}

Financial assistance from the University of Grants Commission (UGC)-Center of Advanced Studies and the Department of Science and Technology-Promotion of University Research and Scientific Excellence programmes, of the Department of Chemistry is gratefully acknowledged. The authors also acknowledge the DST-FIST programme to the Department of Chemistry, Jadavpur University for providing the NMR spectral data. CG and RM are thankful to UGC, New Delhi for their Research Fellowships.

\section{References}

1. Trost B M and Keeley D E 1975 J. Org. Chem. 402013

2. Fujita E and Nagao Y 1977 Bioorg. Chem. 6287

3. Xu R, Cole D, Asberom T, Bara T, Bennett C, Burnett D A, Clader J, Domalski M, Greenlee W, Hyde L, Josien H, Li H, McBriar M, McKittrick B, McPhail A T, Pissarnitski D, Qiang L, Rajagopalan M, Sasikumar T, Su D, Tang H, Wua W-L, Zhang L and Zhao Z 2010 Bioorg. Med. Chem. Lett. 202591

4. Saito M, Nakajima M and Hashimoto S 2000 Tetrahedron 569589

5. Bandini M, Cozzi P G, Giacomini M, Melchiorre P, Selva S and Umani-Ronchi A 2002 J. Org. Chem. 67 3700

6. Garg S K, Kumar R and Chakraborti A K 2005 Synlett 9 1370

7. Chu C M, Gao S, Sastry M N V and Yao C-F 2005 Tetrahedron Lett. 464971

8. Yerli G, Gezegen H and Ceylan M 2012 Org. Commun. 570

9. Srivastava N and Banik B K 2003 J. Org. Chem. 68 2109

10. Chen C-T, Lin Y-D and Liu C-Y 2009 Tetrahedron 65 10470

11. Bakuzis P and Bakuzis M L F 1981 J. Org. Chem. 46 235
12. Cherkauskas J P and Cohen T 1992 J. Org. Chem. 576

13. Li H, Zu L, Xie H, Wang J, Jiang W and Wang W 2007 Org. Lett. 91833

14. Helder R, Arends R, Bolt W, Hiemstra H and Wynberg H 1977 Tetrahedron Lett. 252181

15. Skarzéwski J, Zielinśka-Błajet M and Turowska-Tyrk I 2001 Tetrahedron: Asymmetry 121923

16. McDaid P, Chen Y and Deng L 2002 Angew. Chem. 41 338

17. Emori E, Arai T, Sasai H and Shibasaki M 1998 J. Am. Chem. Soc. 1204043

18. Kanemasa S, Oderaotoshi Y and Wada E 1999 J. Am. Chem. Soc. 1218675

19. Yadav J S, Reddy B V S and Baishya G 2003 J. Org. Chem. 687098

20. Ranu B C, Dey S S and Hajra A 2003 Tetrahedron 59 2417

21. Ranu B C and Dey S S 2004 Tetrahedron 604183

22. Mečirová M, Ťoma S and Kotrusz P 2006 Org. Biomol. Chem. 41420

23. Khan A T, Ghosh S and Choudhury L H 2006 Eur. J. Org. Chem. 712226

24. Khatik G L, Sharma G, Kumar R and Chakraborti A K 2007 Tetrahedron 631200

25. Lenardão E J, Ferreira P C, Jacob R G, Perina G and Leite F P L 2007 Tetrahedron Lett. 486763

26. Banerjee S, Das J, Alvareza R P and Santra S 2010 New J. Chem. 34302

27. Abrouki Y, Zahouily M, Rayadh A, Bahlaouan B and Sebti S 2002 Tetrahedron Lett. 437729

28. Zahouily M, Abrouki Y and Rayadh A 2002 Tetrahedron Lett. 438951

29. Kamal A, Reddy D R and Rajendar A 2005 Tetrahedron Lett. 467951

30. Ito A, Konishi K and Aida T 1996 Tetrahedron Lett. 37 2585

31. Skarzewski J, Zielińska-Błajet M and Turowska-Tyrk I 2003 Tetrahedron 593621

32. Guha C, Pal R and Mallik A K 2012 Arkivoc ix 85

33. Parsons P, Penkett C S and Shell A 1996 J. Chem Rev. 96195

34. Tietze L F, Brasche G and Gericke K M 2006 Domino reactions in organic synthesis (Weinheim: Wiley-VCH)

35. Kumar A and Akanksha 2007 Tetrahedron Lett. 48 8730

36. Abaee M S, Cheraghi S, Navidipoor S, Mojtahedi M M and Forghani S 2012 Tetrahedron Lett. 534405

37. Rao H S P and Jothilingam S 2005 J. Chem. Sci. 117323

38. Jayapal M R and Sreedhar N Y 2010 J. Pharm. Sci. Res. 2644

39. Tiwari V, Ali P and Meshram J 2010 Int. J. ChemTech Res. 21031

40. Pal R, Mandal T K, Guha C and Mallik A K 2011 J. Indian Chem. Soc. $\mathbf{8 8} 711$ 